\title{
Numerical studies of fluid flow across a cosmo ball by using CFD
}

\begin{abstract}
Cosmo ball is a media used in wastewater treatment to increase the surface area of contact and provide longer contact time for biological activities. It is usually placed as a media or as packing in the aeration tank, BioFil tank or even in the activated sludge tank. It induces the growth of bacteria on its surface, which will serve as a contact area for the wastewater and the microbial. The objective of this simulation is to analyze the flow pattern around the Cosmo ball which is been packed in the wastewater treatment tank. By conducting this analysis, the overall insights on the interaction of the fluid flow with the Cosmo ball surface can be visualize. The velocity of the fluid flow across the ball is at a speed of $2 \mathrm{~cm} \mathrm{sec-1}$. The velocity was obtained from the conventional wastewater treatment plant. During the simulation process, the model will undergo each section of program in ANSYS ${ }^{\mathrm{TM}}$ Computational Fluid Dynamics (CFD) such as Design Modeler, Meshing, Pre-Processing, Solver and Post-Processing. The flow distribution across the Cosmo ball was analyzed by varying the angle of attack of the flow. The rationale for this simulation arrangement is that the ball could take any orientation in the bulk media and the fluid flow across it would be at random angle. The result shows that the flow around the Cosmo ball was dispersing sideways thus inducing mixing in the wastewater system. Retention of flow has been observed to occur in the Cosmo ball. The retention of flow provides better microbial interaction with the wastewater.
\end{abstract}

Keyword: Cosmo ball; Computational fluid dynamics; Wastewater; BioFil; Flow; Velocity 